\title{
A GENERAL THEORY OF SUBJECTIVE PROBABILITIES AND EXPECTED UTILITIES
}

\author{
By Peter C. Fishburn \\ Research Analysis Corporation
}

1. Introduction. The purpose of this paper is to present a general theory for the usual subjective expected utility model for decision under uncertainty. With a set $S$ of states of the world and a set $X$ of consequences let $F$ be a set of functions on $S$ to $X . F$ is the set of acts. Under a set of axioms based on extraneous measurement probabilities, a device that is used by Rubin [14], Chernoff [3], Luce and Raiffa [9, Ch. 13], Anscombe and Aumann [1], Pratt, Raiffa, and Schlaifer [11], Arrow [2], and Fishburn [5], we shall prove that there is a realvalued function $u$ on $X$ and a finitely-additive probability measure $P^{*}$ on the set of all subsets of $S$ such that, for all $f, g \varepsilon F$,

$$
f \leqslant g \text { if and only if } E\left[u(f(s)), P^{*}\right] \leqq E\left[u(g(s)), P^{*}\right] .
$$

In $(1), \leqslant$ ("is not preferred to") is the decision-maker's binary preferenceindifference relation and $E(y, z)$ is the mathematical expectation of $y$ with respect to the probability measure $z$.

Because we shall use extraneous measurement probabilities, (1) will be extracted from the more involved expression (2) that is presented in the next section. The axioms we shall use to derive (2) imply that $P^{*}$ is uniquely determined and that $u$ is unique up to a positive linear transformation. $u$ may or may not be bounded: however, it is bounded if there is a denumerable partition of $S$ each element of which has positive probability under $P^{*}$. Our theory places no restrictions on $S$ and $X$ except that they be nonempty sets with $X$ containing at least two elements. $X$ may or may not have a least (most) preferred consequence. In addition, no special restrictions are placed on $P^{*}$. For example, if $S$ is infinite, it may or may not be true that $P^{*}(A)=1$ for some finite subset $A \subseteq S$, and if $P^{*}(A)<1$ for every finite $A \subseteq S$ it may or may not be true that $S$ can be partitioned into an arbitrary finite number $n$ of subsets such that $P^{*}=1 / n$ for each subset. Finally, no special properties will be implied for $u$ apart from its uniqueness and its boundedness in the case noted above.

To indicate briefly how this differs from other theories, we note first that the theories of Chernoff [3], Luce and Raiffa [9], Anscombe and Aumann [1], Pratt, Raiffa, and Schlaifer [11], and Fishburn [5] assume that $S$ is finite. The theory presented here is a generalization of a theory in Fishburn [5]. The theory of Davidson and Suppes [4] assumes that $X$ is finite and implies that, if $x<y$ and $z \prec w$ and there is no consequence between $x$ and $y$ or between $z$ and $w$ then $u(y)-u(x)=u(w)-u(z)$. The theories of Ramsey [13] and Suppes [16]

Received 19 August 1968; revised 6 March 1969. 
place no special restrictions on $S$ but they imply that $X$ is infinite and that if $u(x)<u(y)$ then there is a $z \varepsilon X$ such that $u(z)=.5 u(x)+.5 u(y)$. On the other hand Savage [15] does not restrict $X$ in any unusual way, but his theory requires $S$ to be infinite and implies that, for any positive integer $n$, there is an $n$-part partition of $S$ such that $P^{*}=1 / n$ on each part of the partition. Arrow [2] also assumes this property for $P^{*}$.

2. Definitions and notation. $\beta$ is the set of all simple probability measures (gambles) on $X$, so that if $\mathrm{P} \varepsilon \odot$ then $P(Y)=1$ for some finite $Y$ included in $X$. The probabilities used in $\odot$ are extraneous measurement probabilities. They can be associated with outcomes of chance devices such as dice and roulette wheels.

With $P, Q \varepsilon \rho$ and $\alpha \varepsilon[0,1], \alpha P+(1-\alpha) Q$ is the direct linear combination of $P$ and $Q$ so that $\alpha P+(1-\alpha) Q$ is in $P$. Under this interpretation, $\beta$ is a mixture set. By Herstein and Milnor's [7] definition, a mixture set is a set $M$ and an operation that assigns an element $\alpha a+(1-\alpha) b$ in $M$ to $(a, b) \varepsilon M \times M$ and $\alpha \varepsilon[0,1]$ in such a way that

$$
\begin{aligned}
(1) a+(0) b & =a \\
\alpha a+(1-\alpha) b & =(1-\alpha) b+\alpha a \\
\alpha(\beta a+(1-\beta) b)+(1-\alpha) b & =(\alpha \beta) a+(1-\alpha \beta) b
\end{aligned}
$$

for all $a, b \varepsilon M$ and $\alpha, \beta \varepsilon[0,1]$.

$\mathcal{H}$ is the set of all functions on $S$ to $\mathcal{P}$. With $\boldsymbol{P} \varepsilon \mathcal{H}, \boldsymbol{P}(s)$ is the gamble in $\mathcal{P}$ assigned to $s \varepsilon S$ by $\boldsymbol{P}$. We shall call $\mathfrak{F}$ the set of horse lotteries, after Anscombe and Aumann [1]. A pseudo-operational interpretation for $\boldsymbol{P}$ goes as follows. If $\boldsymbol{P}$ is selected and if state $s$ obtains (to use Savage's term) then the gamble $\boldsymbol{P}(s)$ is used to select a consequence in $X$.

With $P, Q \varepsilon \mathfrak{H C}$ and $\alpha \varepsilon[0,1], \alpha \boldsymbol{P}+(1-\alpha) \boldsymbol{Q}$ is the direct linear combination of $\boldsymbol{P}$ and $\boldsymbol{Q}$ so that $(\alpha \boldsymbol{P}+(1-\alpha) \boldsymbol{Q})(s)=\alpha \boldsymbol{P}(s)+(1-\alpha) \boldsymbol{Q}(s)$. Clearly, $\alpha \boldsymbol{P}+(1-\alpha) \boldsymbol{Q}$ is in $\mathfrak{H C}$ when $\boldsymbol{P}, \boldsymbol{Q} \varepsilon \mathcal{H C}$ and $\alpha \varepsilon[0,1]$. It follows easily that $\mathcal{H}$ is a mixture set.

An event is any subset of $S$. A partition of $S$ is a set of non-empty, disjoint events whose union equals $S . P$ in $\mathcal{H}$ is constant on event $A$ if and only if $\boldsymbol{P}(s)=\boldsymbol{P}\left(s^{\prime}\right)$ for all $s, s^{\prime} \varepsilon A$, in which case we write $\boldsymbol{P}=P$ on $A$ when $\boldsymbol{P}(s)=P$ for all $s \in A$. $P$ and $Q$ agree on event $A$ if and only if $\boldsymbol{P}(s)=\boldsymbol{Q}(s)$ for all $s \varepsilon A$, in which case we write $\boldsymbol{P}=\boldsymbol{Q}$ on $A$.

We shall apply the binary relation $\leqslant$ to $\mathcal{H}$. $P \sim Q$ if and only if $P \leqslant Q$ and $Q \leqslant P$, and $P<Q$ if and only if $P \leqslant Q$ and not $Q \leqslant P$. With $P, Q \varepsilon P$, we define $\leqslant$ on $P$ in terms of $\leqslant$ on $\mathcal{H C}$ thus:

$$
P \leqslant Q \text { if and only if } P \leqslant Q \text { when } P=P \text { and } Q=Q \text { on } S \text {. }
$$

With $P \varepsilon P$ and $Q \varepsilon \mathcal{H}, P \leqslant Q$ means that $P \leqslant Q$ when $P=P$ on $S . P \leqslant Q$, $P \prec Q, P \prec Q, \cdots$ are defined in similar ways.

$A^{c}$ is the complement in $S$ of event $A$, so that $A \cap A^{c}=\varnothing$ and $A \cup A^{c}=S$. 
Event $A$ is null if and only if $\boldsymbol{P} \sim Q$ whenever $\boldsymbol{P}, \boldsymbol{Q}$ in Te agree on $A^{c}$.

With these definitions at hand we shall turn to a set of axioms that implies the existence of a real function $u$ on $X$ and a probability measure $P^{*}$ on (the set of all subsets of ) $S$ such that, for all $P, Q \in \mathfrak{K}$,

$$
P \leqslant Q \text { if and only if } E\left[E(u, P(s)), P^{*}\right] \leqq E\left[E(u, Q(s)), P^{*}\right] .
$$

Expression (1) results from (2) on defining $f \leqslant g$ if and only if $P \leqslant Q$ when $\boldsymbol{P}(s)(f(s))=\boldsymbol{Q}(s)(g(s))=1$ for all $s \varepsilon S$. When $\boldsymbol{P}(s)(f(s))=1$ for all $s \varepsilon S$, $E\left[E(u, P(s)), P^{*}\right]=E\left[u(f(s)), P^{*}\right]$.

3. Axioms and summary theorem. In addition to the structural assumptions of the preceding section ( $\beta$ is the set of simple probability measures on $X$, $\mathfrak{F}$ is the set of all functions on $S$ to $P$ ), we shall use the following six axioms. For all $P, Q, R \in \mathcal{H}$ :

A1. $\leqslant$ is a weak order (transitive and connected, or complete) on $\mathfrak{F}$;

A2. If $\boldsymbol{P} \prec \boldsymbol{Q}$ and $\alpha \varepsilon(0,1)$ then $\alpha \boldsymbol{P}+(1-\alpha) \boldsymbol{R} \prec \alpha \boldsymbol{Q}+(1-\alpha) \boldsymbol{R}$;

A3. If $\boldsymbol{P} \prec \boldsymbol{Q}$ and $\boldsymbol{Q} \prec \boldsymbol{R}$ then $\alpha \boldsymbol{P}+(1-\alpha) \boldsymbol{R} \prec \boldsymbol{Q}$ and $\boldsymbol{Q} \prec \beta P+(1-\beta) \boldsymbol{R}$ for some $\alpha, \beta \varepsilon(0,1)$;

A4. $P \prec Q$ for some $P, Q \varepsilon P$;

A5. If event $A$ is not null, if $P=P$ and $Q=Q$ on $A$ and if $P=Q$ on $A^{c}$ then $P \leqslant Q$ if and only if $P \leqslant Q$;

A6. If $\boldsymbol{P}(s) \prec \boldsymbol{R}$ for all $s \varepsilon S$ then $\boldsymbol{P} \leqslant \boldsymbol{R}$, and if $\boldsymbol{R} \prec \boldsymbol{Q}(s)$ for all $s \varepsilon S$ then $R \leqslant Q$.

Axioms A1, A2, and A3 are fairly standard axioms in von Neumann-Morgenstern or Bernoullian expected-utility theory. A2 is an independence or surething axiom, and a defense for its adoption is similar to the defenses made by Friedman and Savage [6], Savage [15], Raiffa [12], and others. In particular, it can be noted that a two-stage procedure can be associated with $\alpha \boldsymbol{P}+(1-\alpha) \boldsymbol{R}$ whereby $\boldsymbol{P}$ is selected with probability $\alpha$ and $R$ is selected with probability $1-\alpha$ at the first stage. If $\boldsymbol{P}$ is selected at the first stage and if $s$ obtains then $\boldsymbol{P}(s)$ is used to select an $x$ at the second stage. A similar remark holds for $R$ and $R(s)$. If $s$ obtains, the total probability that $x$ will result is $\alpha \boldsymbol{P}(s)(x)+(1-\alpha) \boldsymbol{R}(s)(x)$, which equals the probability $(\alpha \boldsymbol{P}(s)+(1-\alpha) \boldsymbol{R}(s))(x)$ assigned to $x$ by $\alpha \boldsymbol{P}+$ $(1-\alpha) R$ when $s$ obtains.

A3 is a typical Archimedean axiom, and A4 is similar to Savage's P5. It forecloses the uninteresting possibility that all consequences are indifferent to each other. A5 in the $F_{C}$ context is similar in intent and structure to Savage's P3, which is part of his sure-thing principle. A5 says that preferences for constant horse lotteries are the same as preferences for corresponding horse lotteries that are constant on nonnull events and agree with one another on the complements of these events.

The final axiom A6 is another aspect of the sure-thing principle. It is similar to Savage's P7 extended to horse lotteries, except that Savage's P7 uses $\leqslant$ where $\prec$ is used in A6. 
One final definition will be needed for our summary theorem. We shall call $P \varepsilon \mathcal{H C}$ bounded if and only if there are real numbers $a$ and $b$ such that

$$
P^{*}\{a \leqq E(u, P(s)) \leqq b\}=1 .
$$

According to common usage, $P^{*}\{\cdots\}$ is an abbreviation for $P^{*}(\{s \mid a \leqq$ $E(u, \boldsymbol{P}(s)) \leqq b\})$.

Summary Theorem. A1-6 imply that there is a finitely additive probability measure $P^{*}$ on the set of all subsets of $S$ and a real function $u$ on $X$ such that (2) holds for all $P, Q \varepsilon$ KC. Moreover, when A1-6 hold and $P^{*}$ and $u$ satisfy (2) for all $\boldsymbol{P}, \boldsymbol{Q} \varepsilon \mathcal{J}$, then:

(a) Every horse lottery is bounded for $P^{*}$ and $u$;

(b) For all $A \subseteq S, P^{*}(A)=0$ if and only if event $A$ is null;

(c) $u$ is bounded if there is a denumerable partition of $S$ such that each event in the partition has positive probability under $P^{*}$;

(d) A probability measure $Q^{*}$ on the set of all subsets of $S$ and a real function $u^{\prime}$ on $X$ satisfy (2) in place of $P^{*}$ and $u$ for all $P, Q \varepsilon$ JC if and only if $Q^{*}=P^{*}$ and there are real numbers $a>0$ and $b$ such that $u^{\prime}(x)=a u(x)+b$ for all $x \varepsilon X$.

4. Theorems. To establish the Summary Theorem we shall consider a series of theorems, several of which will be proved in the ensuing sections. We shall not prove the first theorem since it follows immediately from the expected-utility theorem proved by Jensen [8] supplemented by Theorem 18 in Luce and Suppes [10, p. 288].

Theorem 1. If $M$ is a mixture set and $\leqslant$ on $M$ is a binary relation that satisfies the direct analogues of $\mathrm{A} 1, \mathrm{~A} 2$, and $\mathrm{A} 3$, then there is a real-valued function $w$ on $M$ that satisfies (3) and (4) for all $a, b \varepsilon M$ and $\alpha \varepsilon[0,1]$ and is unique up to $a$ positive linear transformation when it has these two properties:

$$
\begin{gathered}
a \leqslant b \text { if and only if } w(a) \leqq w(b) \\
w(\alpha a+(1-\alpha) b)=\alpha w(a)+(1-\alpha) w(b) .
\end{gathered}
$$

The next theorem, which uses Theorem 1 in its proof, amounts to a restatement of Theorems 2 and 3 in Fishburn [5]. Its proof is similar to the proofs of those theorems and will be omitted.

TheOREM 2. If A1-5 hold and $\left\{B_{1}, \cdots, B_{n}\right\}$ is a finite partition of $S$ then there are nonnegative numbers $P_{B}{ }^{*}\left(B_{1}\right), \cdots, P_{B}{ }^{*}\left(B_{n}\right)$ that sum to one, and there is a real function $u_{B}$ on $X$ such that, for all $P_{i}, Q_{i} \varepsilon \mathcal{P}$, if

$$
P=P_{i} \text { and } Q=Q_{i} \text { on } B_{i} ; \quad i=1, \cdots, n
$$

then

(5) $\boldsymbol{P} \leqslant Q$ if and only if $\sum_{i=1}^{n} E\left(u_{B}, P_{i}\right) P_{B}{ }^{*}\left(B_{i}\right) \leqq \sum_{i=1}^{n} E\left(u_{B}, Q_{i}\right) P_{B}{ }^{*}\left(B_{i}\right)$.

Moreover, when A1-5 hold and $P_{B}{ }^{*}$ and $u_{B}$ have the stated properties then:

(a) $P_{B}^{*}\left(B_{i}\right)=0$ if and only if $B_{i}$ is null;

(b) Nonnegative numbers $Q_{B}{ }^{*}\left(B_{1}\right), \cdots, Q_{B}{ }^{*}\left(B_{n}\right)$ that sum to one and a real 
function $u_{B}{ }^{\prime}$ on $X$ satisfy (5) in place of $P_{B}{ }^{*}$ and $u_{B}$ for all indicated $\boldsymbol{P}$ and $\boldsymbol{Q}$ if and only if ${Q_{B}}^{*}=P_{B}{ }^{*}$ and $u_{B}{ }^{\prime}$ is a positive linear transformation of $u_{B}$.

Using this theorem we shall establish the following part of the Summary Theorem in the next section. With

$\mathfrak{H}_{0}=\{\boldsymbol{P} \mid \boldsymbol{P} \varepsilon \mathcal{F}$ and $\boldsymbol{P}$ is constant on each event in some finite partition of $S\}$, a horse lottery is in $\mathfrak{H}_{0}$ only if it assigns no more than a finite number of $P \varepsilon \mathcal{P}$ to the states in $S$.

ThEOREM 3. A1-5 imply that there is a probability measure $P^{*}$ on $S$ and a real function $u$ on $X$ such that (2) holds for all $P, Q \varepsilon \mathcal{F}_{0}$. Moreover, when A1-5 hold and $P^{*}$ and $u$ satisfy (2) for all $\boldsymbol{P}, \boldsymbol{Q} \varepsilon \mathfrak{F}_{0}$, then:

(a) For all $A \subseteq S, P^{*}(A)=0$ if and only if event $A$ is null;

(b) A probability measure $Q^{*}$ on $S$ and a real function $u^{\prime}$ on $X$ satisfy (2) in place of $P^{*}$ and $u$ for all $P, Q \varepsilon \mathcal{H}_{0}$ if and only if $Q^{*}=P^{*}$ and $u^{\prime}$ is a positive linear transformation of $u$.

Henceforth in this section $u$ and $P^{*}$ are assumed to satisfy the conditions of Theorem 3. Thus far we have not needed A6, which is required for the next result, to be proved in Section 6 .

Theorem 4. A1-6 imply that (2) holds on the set of all bounded horse lotteries.

We shall prove that all horse lotteries are bounded in two steps, the first of which is accomplished by

Theorem 5. If A1-6 hold and there is a denumerable partition of $S$ such that each event in the partition has positive probability under $P^{*}$, then $u$ on $X$ is bounded.

Clearly, if $u$ on $X$ is bounded then all horse lotteries are bounded. Theorem 5 will be proved in Section 7. To complete the second step of the proof that all horse lotteries are bounded, we consider first the following

Proposition 1. For each positive integer $n$ there is an n-event partition of $S$ such that each event in the partition has positive probability under $P^{*}$.

If Proposition 1 is true then there is a denumerable partition of $S$ for which each event has positive probability. This is probably a well-known fact and we shall omit an explicit proof. The second step for horse-lottery boundedness and the final step in our proof of the Summary Theorem is noted in the following theorem, to be proved in Section 8 .

Theorem 6. If A1-6 hold and if Proposition 1 is false then all horse lotteries are bounded.

5. Proof of Theorem 3. Let A1-5 hold. By considering constant horse lotteries in $\mathcal{H}$, it follows from (5) that if $P, Q \varepsilon \mathcal{P}$ and if $\left\{B_{1}, \cdots, B_{n}\right\}$ and $\left\{C_{1}, \cdots, C_{m}\right\}$ are partitions of $S$ then

$$
E\left(u_{B}, P\right) \leqq E\left(u_{B}, Q\right) \text { if and only if } E\left(u_{C}, P\right) \leqq E\left(u_{C}, Q\right) .
$$

Since $P$ is a mixture set and A1, A2, and A3 imply that analogues of these axioms hold on $P$ (or the set of constant horse lotteries), it follows from Theorem 1 that $u_{C}$ on $X$ is a positive linear transformation of $u_{B}$. Since a positive linear transformation of $u_{B}$ in (5) will not affect the validity of (5), we can therefore 
delete the partition-specific subscript on $u$ and have, in place of (5) when $\boldsymbol{P}=P_{i}$ and $\boldsymbol{Q}=Q_{i}$ on $B_{i}$,

(6) $P \leqslant Q$ if and only if $\sum_{i=1}^{n} E\left(u, P_{i}\right) P_{B}{ }^{*}\left(B_{i}\right) \leqq \sum_{i=1}^{n} E\left(u, Q_{i}\right) P_{B}{ }^{*}\left(B_{i}\right)$.

For an event $A \subseteq S$ let

$$
\mathfrak{H}_{\boldsymbol{A}}=\left\{\boldsymbol{P} \mid \boldsymbol{P} \varepsilon \mathcal{H C} \text { and } \boldsymbol{P} \text { is constant on } A \text { and on } A^{c}\right\} .
$$

$\mathcal{F}_{A}$ is a mixture set. Suppose each of partitions $\left\{B_{1}, \cdots, B_{n}\right\}$ and $\left\{C_{1}, \cdots, C_{m}\right\}$ contains $A$. Then, if $P$ in $\mathcal{H}_{A}$ equals $P_{A}$ on $A$ and $P_{A}{ }^{c}$ on $A^{c},(6)$ implies that, for all $P, Q \varepsilon \mathfrak{H}_{A}$,

$$
\begin{aligned}
P_{B}{ }^{*}(A) E\left(u, P_{A}\right)+\left[1-P_{B}{ }^{*}(A)\right] E\left(u, P_{A}{ }^{c}\right) & \\
& \leqq P_{B}{ }^{*}(A) E\left(u, Q_{A}\right)+\left[1-P_{B}{ }^{*}(A)\right] E\left(u, Q_{A}{ }^{c}\right)
\end{aligned}
$$

if and only if

$$
\begin{aligned}
P_{C}{ }^{*}(A) E\left(u, P_{A}\right)+\left[1-P_{C}{ }^{*}(A)\right] E\left(u, P_{A}{ }^{c}\right) & \\
& \leqq P_{C}{ }^{*}(A) E\left(u, Q_{A}\right)+\left[1-P_{C}{ }^{*}(A)\right] E\left(u, Q_{A}{ }^{c}\right) .
\end{aligned}
$$

It then follows easily from Theorem 1 that $P_{B}{ }^{*}(A)=P_{C}{ }^{*}(A)$. Hence we can drop the partition-specific subscript on $P^{*}$ and rewrite $(6)$ as

(7) $P \leqslant Q$ if and only if $\sum_{i=1}^{n} E\left(u, P_{i}\right) P^{*}\left(B_{i}\right) \leqq \sum_{i=1}^{n} E\left(u, Q_{i}\right) P^{*}\left(B_{i}\right)$.

It follows directly from Theorem 2 that $P^{*}$ is uniquely determined, that $P^{*}(A)=0$ only if $A$ is null, and that $u$ is unique up to a positive linear trans.* formation. Finite additivity for $P^{*}$ is easily demonstrated using partitions $\left\{A, B,(A \cup B)^{c}\right\}$ and $\left\{A \cup B,(A \cup B)^{c}\right\}$ in an analysis like that leading to (7) with $A \cap B=\varnothing$.

Finally, to obtain (2) for all $\boldsymbol{P}, \boldsymbol{Q} \varepsilon \mathcal{F}_{0}$, let $\boldsymbol{P}=P_{i}$ on $B_{i}$ and $\boldsymbol{Q}=Q_{j}$ on $C_{j}$ for the partitions $\left\{B_{1}, \cdots, B_{n}\right\}$ and $\left\{C_{1}, \cdots, C_{m}\right\}$. Applying $(7)$ to the partition

$$
\left\{B_{i} \cap C_{j} \mid i=1, \cdots, n ; j=1, \cdots, m ; B_{i} \cap C_{j} \neq \varnothing\right\}
$$

we obtain

$\boldsymbol{P} \leqslant Q$ if and only if $\sum_{i} \sum_{j} E\left(u, P_{i}\right) P^{*}\left(B_{i} \cap C_{j}\right)$

$$
\leqq \sum_{i} \sum_{j} E\left(u, Q_{j}\right) P^{*}\left(B_{i} \cap C_{j}\right)
$$

which, by finite additivity for $P^{*}$, is the same as

$$
P \preccurlyeq Q \text { if and only if } \sum_{i} E\left(u, P_{i}\right) P^{*}\left(B_{i}\right) \leqq \sum_{j} E\left(u, Q_{j}\right) P^{*}\left(C_{j}\right) .
$$

6. Proof of Theorem 4. Since $\mathcal{F}$ is a mixture set, Theorem 1 implies that there is a real function $v$ on $\mathcal{H}$ such that, for all $P, Q \varepsilon$ TC and $\alpha \varepsilon[0,1]$,

$$
\begin{aligned}
& \boldsymbol{P} \leqslant \boldsymbol{Q} \text { if and only if } v(\boldsymbol{P}) \leqq v(\boldsymbol{Q}) \\
& v(\alpha \boldsymbol{P}+(1-\alpha) \boldsymbol{Q})=\alpha v(\boldsymbol{P})+(1-\alpha) v(\boldsymbol{Q}) .
\end{aligned}
$$


Expressions (8) and (9) hold also for all $P, Q$ in $\mathcal{F}_{0}$. Letting

$$
w(\boldsymbol{P})=E\left[E(u, P(s)), P^{*}\right] \text { for all } \boldsymbol{P} \varepsilon \mathfrak{H}_{0}
$$

it follows from Theorem 3 and

$$
\begin{aligned}
E[E(u, \alpha \boldsymbol{P}(s)+(1-\alpha) & \left.\boldsymbol{Q}(s)), P^{*}\right] \\
= & E\left[\alpha E(u, P(s))+(1-\alpha) E(u, Q(s)), P^{*}\right] \\
= & \alpha E\left[E(u, P(s)), P^{*}\right]+(1-\alpha) E\left[E(u, Q(s)), P^{*}\right]
\end{aligned}
$$

that, for all $P, Q \varepsilon \mathfrak{F}_{0}$ and $\alpha \varepsilon[0,1]$,

$$
\begin{aligned}
& \boldsymbol{P} \leqslant \boldsymbol{Q} \text { if and only if } w(\boldsymbol{P}) \leqq w(\boldsymbol{Q}) \\
& w(\alpha \boldsymbol{P}+(1-\alpha) \boldsymbol{Q})=\alpha w(\boldsymbol{P})+(1-\alpha) w(\boldsymbol{Q}) .
\end{aligned}
$$

Then, by Theorem $1, w$ on $\mathcal{F}_{0}$ is a positive linear transformation of the restriction of $v$ on $\mathfrak{F}_{0}$. By an appropriate transformation we can, with no loss in generality, specify that

$$
v(\boldsymbol{P})=E\left[E(u, \boldsymbol{P}(s)), P^{*}\right]
$$

for all $\boldsymbol{P} \varepsilon \mathfrak{K}_{0}$. According to (8) the proof of Theorem 4 can be completed by proving that (10) holds for all bounded horse lotteries.

Our first step in this direction will be to prove that if $P^{*}(A)=1$ and if $c$ and $d$ defined in the following expression are finite then

(11) $c=\inf \{E(u, \boldsymbol{P}(s)) \mid s \varepsilon A\} \leqq v(\boldsymbol{P}) \leqq \sup \{E(u, \boldsymbol{P}(s)) \mid s \varepsilon A\}=d_{\text {d }}$

Let $\boldsymbol{Q}=\boldsymbol{P}$ on $A$ and $c \leqq \boldsymbol{Q}(s) \leqq d$ on $A^{c}$. Since $A^{c}$ is null, $\boldsymbol{Q} \sim \boldsymbol{P}$ and hence $v(\boldsymbol{P})=v(\boldsymbol{Q})$ by (8). To show that $c \leqq v(\boldsymbol{Q}) \leqq d$ when $c$ and $d$ are finite suppose to the contrary that $d<v(Q)$. With $c \leqq E\left(u, Q^{\prime}\right) \leqq d$ and $Q^{\prime}=Q^{\prime}$ on $S$ let $\boldsymbol{R}=\alpha Q+(1-\alpha) Q^{\prime}$ with $\alpha<1$ near enough to one so that

$$
d<v(\boldsymbol{R})=\alpha v(\boldsymbol{Q})+(1-\alpha) v\left(\boldsymbol{Q}^{\prime}\right)<v(\boldsymbol{Q}) .
$$

Then $R \prec Q$ by (8). But since $E(u, Q(s)) \leqq d<v(R)$ it follows from (8) that $\boldsymbol{Q}(s) \prec R$ for all $s \varepsilon S$ so that axiom $A 6$ implies $Q \leqslant R$, a contradiction. Hence $d<v(\boldsymbol{Q})$ is false. By a symmetric proof, $v(\boldsymbol{Q})<c$ is false. Therefore $c \leqq v(\boldsymbol{Q}) \leqq d$.

With $P$ bounded let $A$ with $P^{*}(A)=1$ be an event on which $E(u, P(s))$ is bounded and let $c$ and $d$ be defined as in (11). If $c=d$ then (10) is immediate. Henceforth assume that $c<d$. For notational convenience we shall take

$$
c=0, \quad d=1 .
$$

Let $\boldsymbol{Q}$ be as defined following (11) so that $v(\boldsymbol{Q})=v(\boldsymbol{P})$ and

To prove that

$$
E\left[E(u, Q(s)), P^{*}\right]=E\left[E(u, P(s)), P^{*}\right] .
$$

$$
v(\boldsymbol{Q})=E\left[E(u, Q(s)), P^{*}\right]
$$


let $\left\{A_{1}, \cdots, A_{n}\right\}$ be the partition (ignoring empty sets) of $S$ defined by

$$
\begin{aligned}
& A_{1}=\{s \mid 0 \leqq E(u, Q(s)) \leqq 1 / n\} \\
& A_{i}=\{s \mid(i-1) / n<E(u, Q(s)) \leqq i / n\} \quad i=2, \cdots, n .
\end{aligned}
$$

Let $P_{i} \varepsilon \rho$ be such that

$$
(i-1) / n \leqq E\left(u, P_{i}\right) \leqq i / n \text { for } i=1, \cdots, n .
$$

The existence of such $P_{i}$ is guaranteed by (11). Then let

$$
\begin{aligned}
\boldsymbol{P}_{i} & =\left\{\begin{array}{lll}
\boldsymbol{Q} & \text { on } & A_{i} \\
P_{i} & \text { on } & A_{i}{ }^{c}
\end{array} \quad i=1, \cdots, n\right. \\
\boldsymbol{P}_{0} & =\sum_{i=1}^{n} n^{-1} \boldsymbol{P}_{i} \\
\boldsymbol{R} & =\sum_{j \neq i}(n-1)^{-1} P_{j} \text { on } A_{i} \text { for } i=1, \cdots, n .
\end{aligned}
$$

Since $\boldsymbol{P}_{0}(s)=\sum_{i} n^{-1} \boldsymbol{P}_{i}(s)=n^{-1} \boldsymbol{Q}(s)+(n-1) n^{-1} \sum_{j \neq i}(n-1)^{-1} P_{j}$ when $s \varepsilon A_{i}$,

$$
P_{0}=n^{-1} Q+(n-1) n^{-1} R,
$$

so that, by (9) and (14),

$$
v(\boldsymbol{Q})=\sum_{i=1}^{n} v\left(\boldsymbol{P}_{i}\right)-(n-1) v(\boldsymbol{R}) .
$$

Since $R \in \mathcal{F}_{0},(10)$ implies that

$$
\begin{aligned}
v(\boldsymbol{R}) & =\sum_{i=1}^{n} E\left(u, \sum_{j \neq i}(n-1)^{-1} P_{j}\right) P^{*}\left(A_{i}\right) \\
& =(n-1)^{-1} \sum_{i=1}^{n}\left[\sum_{j \neq i} E\left(u, P_{j}\right)\right] P^{*}\left(A_{i}\right) .
\end{aligned}
$$

Substituting this in (16) we have

$$
v(\boldsymbol{Q})=\sum_{i=1}^{n} v\left(\boldsymbol{P}_{i}\right)-\sum_{i=1}^{n} \sum_{j \neq i} E\left(u, P_{j}\right) P^{*}\left(A_{i}\right) .
$$

By (12), (13), and (11),

$$
(i-1) / n \leqq v\left(\boldsymbol{P}_{i}\right) \leqq i / n \quad i=1, \cdots, n .
$$

This is true regardless of how the $P_{i}$ are selected so long as they satisfy (12). In particular, since $0=\inf \{E(u, Q(s)) \mid s \varepsilon S\}$ and $1=\sup \{E(u, Q(s)) \mid s \varepsilon S\}$, we can select the $P_{i}$ so that either

$$
E\left(u, P_{1}\right)=1 / n, \text { and } E\left(u, P_{i}\right)=(i-1) / n \text { for } i>1
$$

or

(20) $E\left(u, P_{i}\right)=i / n$ for $i<n$, and $E\left(u, P_{n}\right)=(n-1) / n$.

Applying (19) and the left side of $(18)$ to $(17)$ we get

$$
\begin{aligned}
v(Q) \geqq & \sum_{i=1}^{n}(i-1) / n-\frac{1}{2}(n-1) P^{*}\left(A_{1}\right) \\
& -\sum_{i=2}^{n}\left[\frac{1}{2}(n-1)-(i-1) / n+1 / n\right] P^{*}\left(A_{i}\right) \\
= & \frac{1}{2}(n-1)-\frac{1}{2}(n-1)+\sum_{i=2}^{n}(i-1) n^{-1} P^{*}\left(A_{i}\right)-n^{-1}\left[1-P^{*}\left(A_{1}\right)\right] \\
\geqq & \sum_{i=1}^{n}(i-1) n^{-1} P^{*}\left(A_{i}\right)-1 / n .
\end{aligned}
$$


Applying (20) and the right side of (18) to (17) we get

$$
\begin{aligned}
v(Q) \leqq & \sum_{i=1}^{n} i / n-\sum_{i=1}^{n-1}\left[\frac{1}{2}(n-1)-i / n+(n-1) / n\right] P^{*}\left(A_{i}\right) \\
& -\frac{1}{2}(n-1) P^{*}\left(A_{n}\right) \\
= & \sum_{i=1}^{n} i n^{-1} P^{*}\left(A_{i}\right)+n^{-1}\left[1-P^{*}\left(A_{n}\right)\right] \\
\leqq & \sum_{i=1}^{n} i n^{-1} P^{*}\left(A_{i}\right)+1 / n .
\end{aligned}
$$

By the definition of $E, \quad \sum(i-1) n^{-1} P^{*}\left(A_{i}\right) \leqq E\left[E(u, Q(s)), P^{*}\right] \leqq$ $\sum \operatorname{in}^{-1} P^{*}\left(A_{i}\right)$, so that

$$
\left|v(Q)-E\left[E(u, Q(s)), P^{*}\right]\right| \leqq 2 / n \text { for } n=1,2, \cdots .
$$

Therefore, $v(Q)=E\left[E(u, Q(s)), P^{*}\right]$.

7. Proof of Theorem 5. Let A1-6 hold and let $\bar{A}$ be a denumerable partition of $S$ wth $P^{*}(A)>0$ for all $A \varepsilon \bar{A} .\left\{P^{*}(A) \mid A \varepsilon \bar{A}\right\}$ must have a largest element, say $P^{*}\left(A_{1}\right)$. Then $\left\{P^{*}(A) \mid A \varepsilon \bar{A}-\left\{A_{1}\right\}\right\}$ must have a largest element, say $P^{*}\left(A_{2}\right)$. Continuing this process we get a sequence

and

$$
A_{1}, A_{2}, \cdots \text { with }\left\{A_{1}, A_{2}, \cdots\right\}=\bar{A}
$$

(21) $\quad P^{*}\left(A_{1}\right) \geqq P^{*}\left(A_{2}\right) \geqq P^{*}\left(A_{3}\right) \geqq \cdots ; \quad P^{*}\left(A_{i}\right)>0$ for all $i$.

For definiteness suppose that $u$ is unbounded above. By a linear transformation of $u$ we can assume that $[0, \infty) \subseteq\{E(u, P) \mid P \varepsilon \mathcal{P}\}$. Let $P_{i} \varepsilon \mathcal{P}$ and $P \varepsilon \mathcal{H}_{\text {. }}$ be such that

$$
\begin{aligned}
E\left(u, P_{i}\right) & =P^{*}\left(A_{i}\right)^{-1} \quad i=1,2, \cdots \\
P & =P_{i} \quad \text { on } \quad A_{i} \quad i=1,2, \cdots .
\end{aligned}
$$

Also let $Q_{n} \varepsilon \mathcal{H}$ be constant on each $A_{i}(i \leqq n)$ and constant on $\mathrm{U}_{i=n+1}^{\infty} A_{i}$ with

$$
\begin{aligned}
& E\left(u, Q_{n}(s)\right)=P^{*}\left(A_{n}\right)^{-1}-P^{*}\left(A_{i}\right)^{-1} \quad s \varepsilon A_{i} ; \quad i=1, \cdots, n \\
& E\left(u, Q_{n}(s)\right)=0 \quad s \varepsilon \bigcup_{i=n+1}^{\infty} A_{i} .
\end{aligned}
$$

Letting $v$ on $\mathfrak{H C}$ be as given in (8) and (9) and satisfying (10) on $\mathfrak{H}_{0}$,

$$
\begin{aligned}
v\left(Q_{n}\right) & =\sum_{i=1}^{n}\left[P^{*}\left(A_{n}\right)^{-1}-P^{*}\left(A_{i}\right)^{-1}\right] P^{*}\left(A_{i}\right) \\
& =P^{*}\left(A_{n}\right)^{-1} \sum_{i=1}^{n} P^{*}\left(A_{i}\right)-n \text { for } n=1,2, \cdots
\end{aligned}
$$

since $Q_{n} \varepsilon \mathfrak{F}_{0}$ for each $n$.

By (22) and (23),

$$
\begin{aligned}
E\left(u, \frac{1}{2} P(s)+\frac{1}{2} Q_{n}(s)\right) & =\frac{1}{2} P^{*}\left(A_{i}\right)^{-1}+\frac{1}{2}\left[P^{*}\left(A_{n}\right)^{-1}-P^{*}\left(A_{i}\right)^{-1}\right] \\
& =\frac{1}{2} P^{*}\left(A_{n}\right)^{-1} \text { for all } s \varepsilon \bigcup_{1}^{n} A_{i}
\end{aligned}
$$

and, by (21) and (23),

$$
E\left(u, \frac{1}{2} P(s)+\frac{1}{2} Q_{n}(s)\right) \geqq \frac{1}{2} P^{*}\left(A_{n}\right)^{-1} \text { for all } s \varepsilon \mathrm{U}_{n+1}^{\infty} A_{i} .
$$


Therefore

$$
\frac{1}{2} P^{*}\left(A_{n}\right)^{-1}=\inf \left\{E\left(u, \frac{1}{2} P(s)+\frac{1}{2} Q_{n}(s)\right) \mid s \varepsilon S\right\},
$$

and hence, by (11), $v\left(\frac{1}{2} P+\frac{1}{2} Q_{n}\right) \geqq \frac{1}{2} P^{*}\left(A_{n}\right)^{-1}$, which, using (9) and (24) implies that

$$
\begin{aligned}
v(\boldsymbol{P}) & \geqq P^{*}\left(A_{n}\right)^{-1}-P^{*}\left(A_{n}\right)^{-1} \sum_{i=1}^{n} P^{*}\left(A_{i}\right)+n \\
& \geqq n \quad \text { for } \quad n=1,2, \cdots .
\end{aligned}
$$

Since this requires $v(\boldsymbol{P})$ to be infinite we have obtained a contradiction and conclude that $u$ is not unbounded above. By a symmetric proof, $u$ is not unbounded below. Hence, the hypotheses of Theorem 5 imply that $u$ is bounded.

8. Proof of Theorem 6. Let A1-6 hold and assume that Proposition 1 is false. Then there must be a (unique) positive $m$ for which there is an $m$-event partition of $S$ that has positive probability for each event and such that every partition of $S$ has at most $m$ events that have positive probability.

For convenience assume that $u(y)=0$ for a $y \varepsilon X$. Suppose then that $Q$ in $\mathfrak{F C}$ is unbounded. For definiteness, assume that $Q$ is unbounded above. Let $\boldsymbol{P}$ be obtained from $Q$ by replacing each $x$ for which $Q(s)(x)>0$ and $u(x)<0$ by $y$ with $u(y)=0$, for all $s \varepsilon S$. Then $E(u, P(s)) \geqq 0$ for all $s \varepsilon S$ and $\boldsymbol{P}$ is unbounded above so that, for every positive integer $n$,

$$
P^{*}\{E(u, P(s)) \geqq n\}>0 .
$$

Because no partition has more than $m$ events with positive probability, $P^{*}\{E(u, \boldsymbol{P}(s)) \geqq n\}$ can change no more than $m$ times as $n$ increases. Hence, there is a positive integer $N$ and an $\alpha>0$ such that

$$
P^{*}\{E(u, P(s)) \geqq n\}=\alpha \text { for all } n \geqq N .
$$

Let

$$
E\left(u, P_{i}\right)=i \text { for } i=1,2, \cdots
$$

and

$$
\begin{aligned}
& \boldsymbol{Q}_{n}=\left\{\begin{array}{l}
\boldsymbol{P} \text { on } \quad\{s \mid E(u, \boldsymbol{P}(s)) \geqq n\} \\
P_{n} \text { on } \quad\{s \mid E(u, \boldsymbol{P}(s))<n\}
\end{array}\right. \\
& \boldsymbol{R}_{n}=\left\{\begin{array}{l}
P_{n} \text { on } \quad\{s \mid E(u, \boldsymbol{P}(s)) \geqq n\} \\
\boldsymbol{P} \text { on }\{s \mid E(u, \boldsymbol{P}(s))<n\} .
\end{array}\right.
\end{aligned}
$$

Then, with $\boldsymbol{P}_{n}=P_{n}$ on $S, \frac{1}{2} \boldsymbol{P}+\frac{1}{2} \boldsymbol{P}_{n}=\frac{1}{2} Q_{n}+\frac{1}{2} \boldsymbol{R}_{n}$, so that with $v$ on $\mathcal{H C}$ as defined by (8) and (9) and satisfying (10) for all bounded horse lotteries,

$$
v(\boldsymbol{P})+n=v\left(\boldsymbol{Q}_{n}\right)+v\left(\boldsymbol{R}_{n}\right) \quad n=1,2, \cdots .
$$

Since $\boldsymbol{R}_{n}$ is bounded, its definition, (10), and (25) imply

$$
v\left(\boldsymbol{R}_{n}\right)=E\left[E\left(u, \boldsymbol{R}_{n}(s)\right), P^{*}\right] \geqq n \alpha \text { for all } n \geqq N .
$$


Since $P_{n-1} \prec Q_{n}(s)$ for all $s \varepsilon S, A 6$ implies that $P_{n-1} \leqslant Q_{n}$ so that

$$
v\left(Q_{n}\right) \geqq n-1 \text { for all } n \text {. }
$$

These inequalities and (26) yield

$$
v(\boldsymbol{P}) \geqq n \alpha-1 \text { for all } n \geqq N,
$$

which requires $v(\boldsymbol{P})$ to be infinite, a contradiction. Therefore $\boldsymbol{Q}$ cannot be unbounded above. A symmetric proof shows that $Q$ cannot be unbounded below. Hence, when $A 1-6$ hold and Proposition 1 is false, every horse lottery is bounded.

\section{REFERENCES}

[1] Anscombe, F. J. and Aumann, R. J. (1963). A definition of subjective probability. Ann. Math. Statist. 34 199-205.

[2] Arrow, K. J. (1966). Exposition of a theory of choice under uncertainty. Synthese $16253-269$.

[3] Chernoff, H. (1954). Rational selection of decision functions. Econometrica 22 422-443.

[4] Davidson, D. and Suppes, P. (1956). A finitistic axiomatization of subjective probability and utility. Econometrica 24 264-275.

[5] Fishburn, P. C. (1967). Preference-based definitions of subjective probability. Ann. Math. Statist. 38 1605-1617.

[6] Friedman, M. and Savage, L. J. (1952). The expected-utility hypothesis and the measurability of utility. J. Political Economy $60463-474$.

[7] Herstein, I. N. and Milnor, J. (1953). An axiomatic approach to measurable utility. Econometrica 21 291-297.

[8] Jensen, N. E.(1967). An introduction to Bernoullian utility theory. I. Utility functions. Swedish J. Economics 69 163-183.

[9] Luce, R. D. and RaIfFa, H. (1957). Games and Decisions. Wiley. New York.

[10] Luce, R. D. and Suppes, P. (1965). Preference, utility, and subjective probability. Handbook of Mathematical Psychology. 3 249-410. Wiley, New York.

[11] Pratt, J. W., Raiffa, H. and Schlaifer, R. (1964). The foundations of decision under uncertainty: an elementary exposition. J. Amer. Statist. Assoc. 59 353-375.

[12] Raiffa, H. (1961). Risk, ambiguity, and the Savage axioms: comment. Quarterly $J$. Economics 75 690-694.

[13] Ramsey, F. P. (1931). The Foundations of Mathematics and Other Logical Essays. Harcourt, Brace, and Co., New York. [Reprinted in H. E. Kyburg and H. E. Smokler (Eds.), Studies in Subjective Probability. Wiley, New York, 1964.]

[14] Rubin, H. (1949). Postulates for the existence of measurable utility and psychological probability (abstract). Bull. Amer. Math. Soc. 55 1050-1051.

[15] Savage, L. J. (1954). The Foundations of Statistics. Wiley, New York.

[16] Suppes, P. (1956). The role of subjective probability and utility in decision making. Proc. Third Berkeley Symp. Math. Statist. Prob. 5 61-73. Univ. of California Press. 